S.D. Ryder, D.J. Pisano, M.A. Walker, and K.C. Freeman, eds.

\title{
Dark Matter in Galaxies: Observational overview
}

\author{
A. Bosma \\ Observatoire de Marseille, 2 Place Le Verrier, 13248 Marseille Cedex 4, \\ France
}

\begin{abstract}
I review the observational side of the present state of the debate about the dark matter in galaxies, with emphasis on the core/cusp problem in low surface brightness galaxies, and the question of maximum / sub-maximum disks in spiral galaxies. Some remarks are made about the dwarf spheroidals around the Milky Way, and about elliptical galaxies.
\end{abstract}

\section{Introduction}

The WMAP results (Spergel et al. 2003) have given rather strict limits on the matter and energy content of the Universe, in agreement with the $\Lambda$ CDM model. Typically, for $\Omega_{\text {total }}=1, \Omega_{\text {dark energy }}=0.73$, and $\Omega_{\text {matter }}=0.27$, with $\Omega_{\text {baryon }}=$ 0.044 , and $\Omega_{\text {non-baryonic }}=0.23$. Some of these results depend on data from the $2 \mathrm{dF}$ Galaxy Redshift Survey. Initial results from the SDSS redshift survey are not much different (Tegmark et al. 2003). The ratio of baryonic to non-baryonic matter is thus 1 to $\sim 5-6$. The baryonic matter at $\mathrm{z}=0$ is mostly not in stars $\left(\Omega_{\text {stars }}=0.005\right)$, and the matter budget in e.g. the Local Group is still not well known : there could be hot gas in substantial amounts (Nicastro et al. 2003).

These results further support the $\Lambda \mathrm{CDM}$ model for structure formation in the Universe, and underscore the need to understand galaxies in the framework of this theory. However, at the scale of galaxies, the "predictions" of the $\Lambda$ CDM model depend on numerical simulations, which, despite their sophistication, suffer from inadequate resolution, and may miss some of the physics. This meeting aims to see how well specific predictions from current cosmological simulations of dark matter halos fare on issues such as their central density profiles, the relation between dark to baryonic matter there, and their shape and extent.

\section{Some issues concerning mass models of spiral galaxies}

Over the last 30 years observations, in particular rotation curves derived from HI data, have clearly established that the "visible" parts of a spiral galaxy, i.e. the stellar bulge and disk, and the HI gas layer, cannot account for the observed high rotation speeds in the outer parts of spirals, if the stellar mass-to-light ratio is constant with radius. This discrepancy is attributed to unseen matter, often taken to be a (spherical) dark halo. Several issues complicate this picture :

Assumptions going into the derivation of the rotation curve. A large fraction of galaxies have a warped HI layer in the outer parts, which is modeled with a 


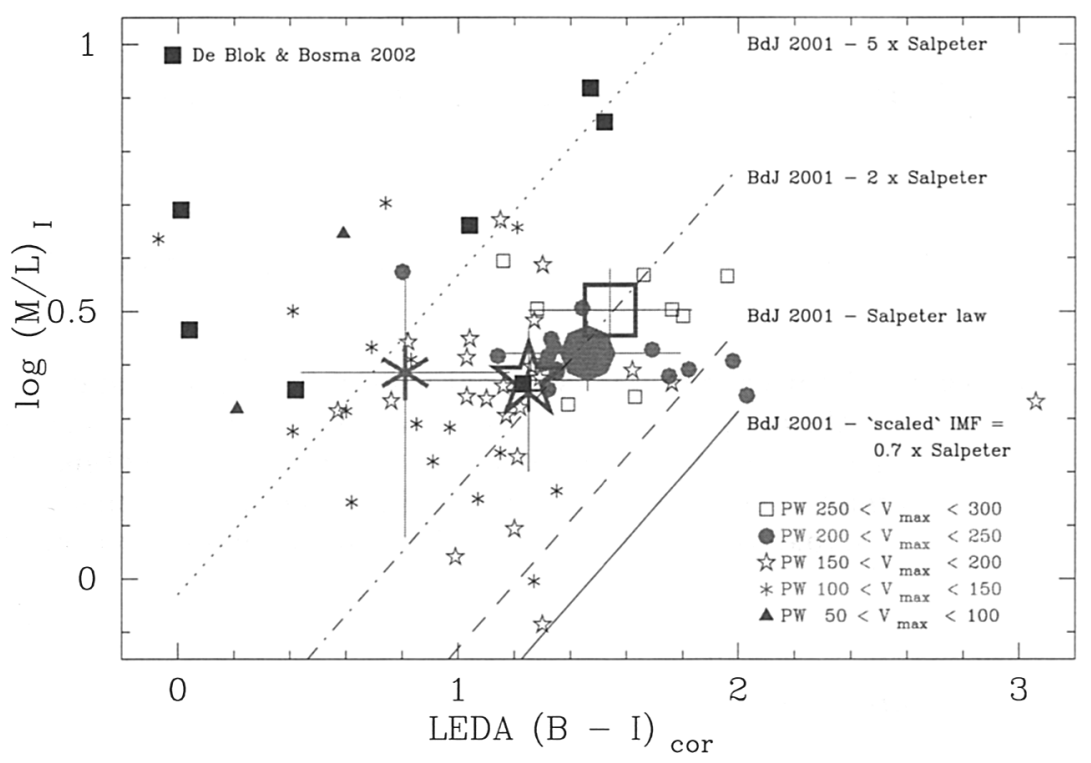

Figure 1. Relation between the mass-to-light ratio in the I-band and the global (B-I) colour corrected for galactic and internal extinction, from the LEDA database, for galaxies in the Palunas \& Williams (2000) sample. Different symbols indicate differences in the maximum rotation velocity $\mathrm{V}_{\max }$. Large symbols represent averages per class of $\mathrm{V}_{\max }$. Various lines indicate models from Bell \& de Jong (2001, table 1), different only in the scaling of the IMF. Black squares come from data of de Blok \& Bosma (2002) for LSB galaxies, rescaled to the I-band.

"tilted ring" model in which the orientation of the rings is gradually changed to allow for the peculiarities of the velocity field. Non-axisymmetric disturbances, such as bars or oval distortions, or peculiar velocities due to spiral structure, are in the first instance ignored, and an azimuthally averaged rotation curve derived. Asymmetries, if small enough, are likewise ignored. All these assumptions may introduce systematic errors in the derived rotation curves at the $5-15 \%$ level.

Values of the mass-to-light ratio are not well determined. This leads to a degeneracy in the mass models. At one extreme, the maximum disk approach tries to fit as much of the mass into the disk as possible, without overshooting the rotation curve. For optical curves, most galaxies can then be fitted without a dark halo, due to the limited extent of the curve. In Figure 1 I plot, for a sample of galaxies studied by Palunas \& Williams $(2000)$, the $\mathrm{M} / \mathrm{L}_{\mathrm{I}}$-ratio they determined from maximum disk fits against the extinction corrected (B-I) colour I collected from the LEDA database. For large galaxies a trend exists: on average redder disks have higher $\mathrm{M} / \mathrm{L}$ ratios, as expected e.g. in models from Bell \& de Jong (2001). Yet there is a fair amount of scatter in each class of galaxies selected according to their maximum rotation velocity $\mathrm{V}_{\max }$, in particular for 
smaller (bluer) galaxies. The required $\mathrm{M} / \mathrm{L}$ values are too high compared to those predicted by Bell \& de Jong's "preferred" Initial Mass Function (IMF), the "scaled IMF" $=0.7 \times$ Salpeter curve, based on maximum disk + halo fits for Ursa Major galaxies (Verheijen 1997). The extreme maximum disk models of the galaxies in the Palunas \& Williams sample require a bottom-heavy IMF.

This problem is aggravated for low surface brightness (LSB) disk galaxies, as is shown by the few points taken from models of such galaxies by de Blok \& Bosma (2002). This clearly illustrates that the dark matter problem in these galaxies is much worse, and has lead to the assumption that even a "minimum $\operatorname{disk"}(\mathrm{M} / \mathrm{L}=0)$ is a reasonable approximation to the real mass distribution.

Scaling the HI can produce a good fit instead of a halo. Bosma (1978) noticed that the surface density ratio of HI gas mass to total mass becomes constant with radius in the outer parts. Reversely, one can thus scale the curve for the HI gas with a certain factor to fit the rotation curve, instead of with a halo. Hoekstra et al. (2001) did this for a number of spirals, and Swaters (1999) for a number of dwarf galaxies. The scaling factor is not constant, and varies with the maximum velocity of a galaxy (cf. Figure 2). For a number of galaxies the fits may well break down at larger radii if more sensitive HI data become available.
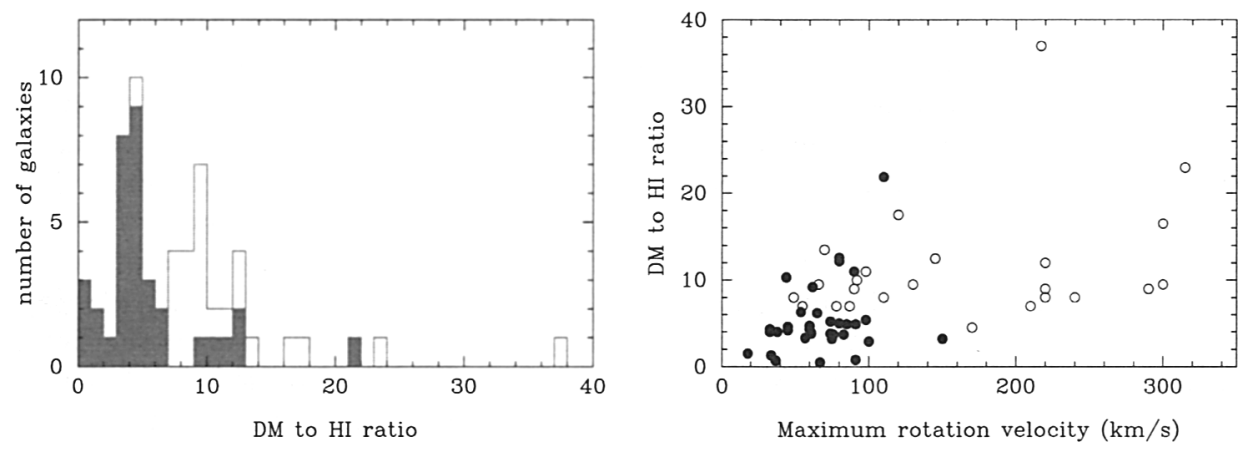

Figure 2. HI component scaled as "dark halo" : a) histogram of the scaling factor, b) scaling factor vs. $V_{\max }$. Based on data from Hoekstra et al. (2001) (unshaded part of histogram, open circles), and Swaters (1999) (shaded part of histogram, filled circles)

\section{The core/cusp problem in LSB galaxies}

Cosmological numerical simulations invariably produce cuspy dark halos, but the precise value of the inner slope $\alpha$ of the radial density profile $\rho \sim \mathrm{r}^{\alpha}$ is debated. Moore et al. (1999) and Fukushige \& Makino (2001) advocate $\alpha=-1.5$, and Navarro, Frenk \& White $(1996,1997)$ an inner slope of -1.0 . Both White and Navarro (this volume) report on work by Hayashi et al. (2003) : there is no real convergence towards a unique value of the inner slope, the resolution prohibits predictions to be made inside a radius of $\sim 1 \mathrm{kpc}$, but the profile remains cuspy, and there is some cosmic scatter (see also Fukushige, Kawai \& Makino 2003). 
This situation differs from an until recently widespread perception that the NFW profile, with inner slope -1.0 , should be considered "universal", and the yardstick against which the observations should be discussed. I will use the slope -1.0 here as a fiducial mark to see where the observations stand, and which is to be improved as the cosmological numerical simulations become more realistic (e.g. by having adequate resolution on the dwarf galaxy scale, and perhaps incorporating more relevant physics), and better understood theoretically (e.g. by demonstrating which physical process, or combination of processes, sets up the value of the inner slope in dark matter dominated galaxies).

\subsection{Inner slope values: technical and selection issues}

Most observers conclude that $\alpha$ in LSB galaxies is closer to 0.0 than to -1.0 , and almost all find that the decomposition mass models work better if the dark halo is modeled with a (pseudo-) isothermal sphere, or a Burkert profile, rather than with the NFW profile. However, some authors maintain that the problem of determining the inner slope is fraught with systematic effects, which all magically contribute towards shallower slopes. The issue debated is not only about the best value for the inner slope, but also whether a slope of -1.0 can be reasonably excluded, so as to force a modification of the $\Lambda$ CDM picture at galaxy scales. See also the contributions by de Blok and by Swaters (this volume).

On the selection side, the debate is about the quality and relevance of the data. As more galaxies are observed, stricter selection criteria can be used to retain only galaxies with sufficiently "good" data. Thus one excludes 1) galaxies with poor angular resolution, thereby requiring in most cases supplementary $\mathrm{H} \alpha$ data in addition to a HI rotation curve, so that the inner $1 \mathrm{kpc}$ of a galaxy is well probed. This excludes far away galaxies or galaxies for which there are only low resolution HI data, and thus minimizes slit width effects; 2) edge-on galaxies, which are apparently too difficult to understand for some workers in the field, despite demonstrations by Bosma et al. (1992) and Matthews \& Wood (2001) that small galaxies seen edge-on are transparent and have rotation curves which are slowly rising in the inner parts. The argument that somehow there is no emission at the tangent point leads to very peculiar $\mathrm{H} \alpha$ distributions in more face-on galaxies which are yet to be seen; 3) galaxies with large asymmetries, faint emission, etc., which have low quality rotation curves.

De Blok, Bosma \& McGaugh (2003) applied these criteria to data of McGaugh et al. (2001) and de Blok \& Bosma (2002), and find for a restricted sample that the values of the inner slope are between 0.2 and -1.0 (see de Blok, this volume). Swaters et al. (2003a), in a similar study, come to a similar conclusion. De Blok et al. (2003) also report about deliberate slit offsets and misalignments for one galaxy, UGC 4325, and conclude that these effects could not magically bring the slope from about -0.2 to -1.0 , although of course scatter is introduced if the major axis is not observed correctly.

Finally, for several galaxies there is more than one observation, done with different telescopes, and by different observers. Even though the error bars are sometimes unsatisfactorily large, there is good agreement in general. The situation for UGC 4325, debated at the meeting, is shown in Figure 3. The HI position-velocity diagram prompts me to think that the peak velocity is indeed around $\sim 110 \mathrm{~km} / \mathrm{s}$ as it is in the optical, after which the rotation curve declines. 


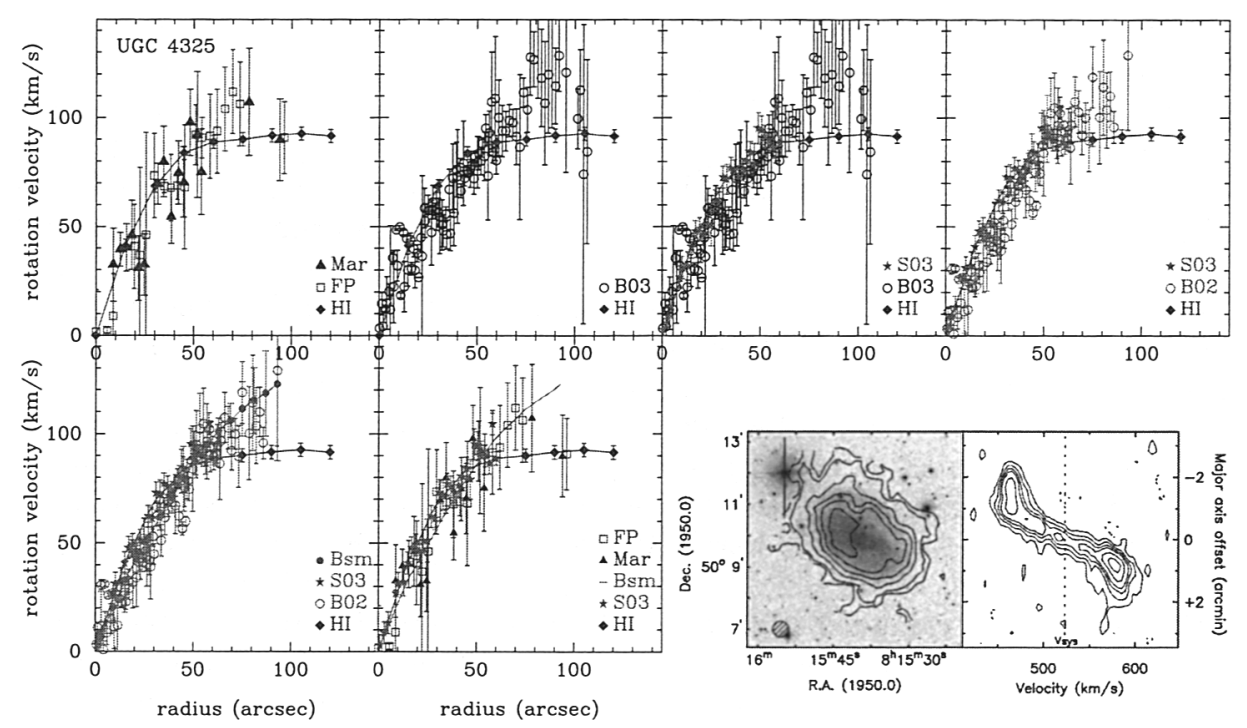

Figure 3. Comparison of data for UGC 4325. HI data from Swaters 1999 (HI), Fabry-Pérot data from Garrido et al. 2002 (FP), long slit data from Marchesini et al. 2002 (Mar), de Blok \& Bosma 2002 (B02), de Blok et al. 2003 (B03), and Swaters et al. 2003a (S03). Bsm represents smoothed long slit data as described in B02. At lower right the HI distribution and position-velocity diagram from Swaters 1999.

\subsection{Inner slope values: astrophysical issues}

The determination of the kinematical center, and the influence of non-circular motions on the rotation curves are also debated.

The rather irregular nature of some of the dwarf or LSB galaxies studied, and the faintness of particularly the near-infrared images makes the determination of the center of such galaxies difficult. Interestingly, HST data on central star clusters in late type spirals is available for a number of the galaxies used in the core/cusp studies, and comparison of these positions and the centers of the inner disks show agreement to within 1-2 arcsec (Böker et al. 2002).

For the galaxy IC 2574, I reanalyzed the HI data obtained by Walter \& Brinks (1999), and deliberately varied the position of the center. In some cases I also carved up the galaxy into annuli, and allowed the center of each annulus to vary with respect to the next one. Despite the variation of the center positions with an r.m.s. scatter of about $250 \mathrm{pc}$, the determined slopes still come out to be $-0.15 \pm 0.38$, well away from the fiducial NFW slope of -1.0 .

Bars could contribute to non-circular motions in the inner parts, and thus lead to a poor quality rotation curve when long slit data are used, or when a two-dimensional velocity field is not properly analysed. Swaters et al. (2003a) assert that bars in their long slit data sample predominantly have shallow slopes, but inspection shows that UGC 2259, UGC 4499, and UGC 5721, as well as F568-3 are also barred, in addition to the galaxies they identify as such. For 
the remaining unbarred galaxies, there is a wide variety of slopes. Swaters et al. (2003b) observed the galaxy DDO 39 with an integral field spectrograph yielding a two-dimensional velocity field, which shows that the inner parts of this galaxy are affected by non-circular motion caused by a bar-like distortion. Several other studies of two-dimensional $\mathrm{H} \alpha$ velocity fields are underway. Garrido et al. (2002) report on data obtained with a Fabry-Pérot instrument, and Bolatto (this meeting, see Simon et al. 2003) report on data obtained with an integral field spectrograph, combined with $\mathrm{CO}$ data obtained with BIMA. The results so far for NGC 4605 and NGC 2976 are that the inner slopes are rather shallow, and that the non-circular motions are mainly in the inner parts.

One can look at bars in LSB galaxies in two ways: 1) they could be similar to bars in HSB galaxies, or 2) they could be just the response to the expected triaxiality of the dark halo. Since the reported non-circular motions are stronger in the inner parts for DDO 39 and NGC 2976, it seems likely that those galaxies behave like HSB's, where the disk is dynamically important in the inner parts. Yet it cannot be excluded that whole disks are elongated due to the triaxial halo. One way to study this further is to look at results of a Fourier analysis of velocity fields of a number of galaxies, performed by Schoenmakers (1999). From his work, I have collected the results on disk elongation in Figure 4a, which shows that the scatter in $\epsilon_{\text {pot }} \sin \phi$ is larger for galaxies with smaller maximum rotation velocity. Thus disks in small galaxies could be globally elongated due to the forcing of a triaxial halo. Interestingly, the small galaxy with no elongation, NGC 247, was declared in van den Bosch et al. (2000) "the only galaxy found [so far] capable of limiting the slope of the dark matter profile". True enough, its slope is -1.02 , close the the NFW slope, but perusal of Schoenmakers' results and a near infrared image show the presence of a bar, and a strong lopsidedness.

In future, the effort will thus shift towards evaluating the importance of the disc component in LSB galaxies, using near-infrared surface photometry, and two-dimensional velocity fields at high spatial resolution. Meanwhile, some evaluation of the importance of non-circular motions can be had by studying bars in HSB galaxies. Disk elongation can be constrained using the Tully-Fisher relation (Franx \& de Zeeuw 1992). Early work on the barred spiral NGC 5383 (Athanassoula 1984) shows that the viewing angle is important : some angles are more favourable than others. Neither the mean rotation curve, nor the positionvelocity cut along the major axis can be trusted as a good representation of the true rotation curve, but the deviations scale with the strength of the bar. For the weak bars in LSB galaxies, it is not obvious how strong these effects are.

\section{The importance of disk self gravity in HSB spirals}

This so-called "maximum disk" problem is related to the core/cusp problem, since in the current $\Lambda \mathrm{CDM}$ picture the dark halo dominates the potential in the central parts also in high surface brightness spirals. Several ways have been explored to break the mass model degeneracy using other dynamical considerations concerning the importance of the disk in the inner parts of spiral galaxies.

Athanassoula et al. (1987, ABP) use swing amplifier criteria, which depend on the rotation curve shape and on a characteristic $\mathrm{X}$ parameter dependent on the epicyclic frequency $\kappa$, the number of arms $\mathrm{m}$, and the active surface mass 

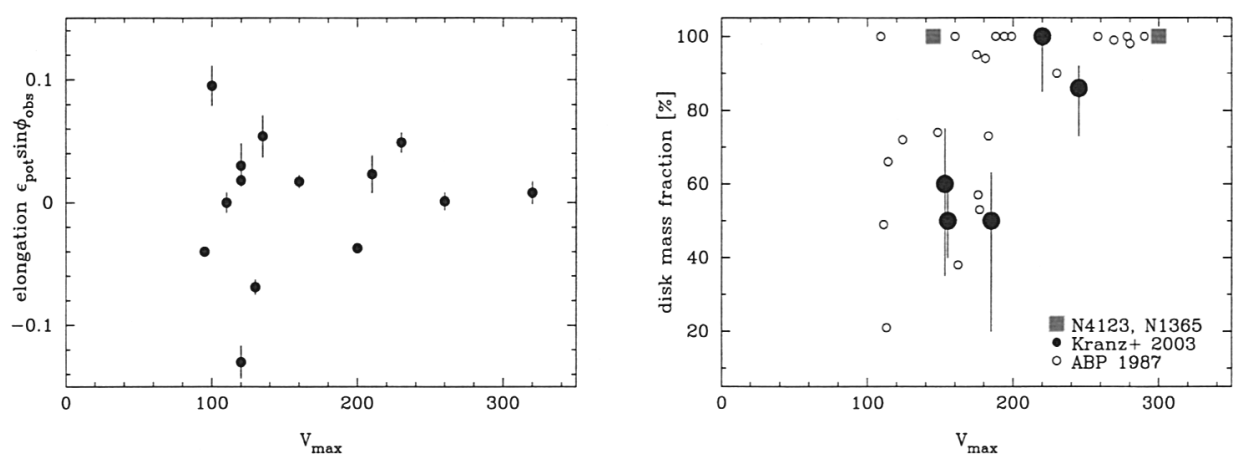

Figure 4. a) Disk elongation against $V_{\max }$, data extracted from Schoenmakers (1999) b) Disk mass fraction against $V_{\max }$, data from Kranz et al. (2003) with N4123 (left) and N1365 added. Faster rotators seem to have rounder disks, which are more self-gravitating.

density of the disk. By requiring that the swing amplification of the $\mathrm{m}=2$ perturbations is possible, the range of mass-to-light ratios is limited to a factor of 2 : a lower limit set by requiring that the disk is massive enough to just allow amplification of the $\mathrm{m}=2$ perturbations, and an upper limit set by requiring that amplification of the $\mathrm{m}=1$ perturbations is just prohibited. Usually the latter condition holds for a model with maximum disk and a non-hollow halo.

Peculiar motions due to the spiral arms were clearly seen in the early M81 $21 \mathrm{~cm}$ line data, and modeled with a spiral density wave response calculation by Visser (1980), who did not include a dark halo in his models. The presence of "wiggles" in position-velocity curves from long slit data are thus associated with the spiral arms. Kranz et al. (2001) use such data for NGC 4254 and try to reproduce the observed velocity perturbations with a stationary gas flow model using the K-band image of this galaxy as input to the evaluation of the disk part of the galactic potential. They find that a maximum disk model produces too large velocity perturbations, and put an upper limit on the disk mass fraction (the mass ratio between a given disk model and the maximum disk model) of 0.8. However, this galaxy is lopsided in the HI, the spiral may be evolving, the small bar in the center of the galaxy might have a different pattern speed than the main spiral pattern, the inclination may be higher than the authors assume, and the adopted method may favour lower disk mass fractions (Slyz et al. 2003). Kranz et al. (2003) report on a similar analysis for four more cases, and find a trend that the brightest spirals (those with the highest rotational velocities), seem to have maximum disks, but that towards lower luminosity spirals the relative influence of the dark matter in the inner parts increases. Comparison with data from ABP shows good agreement with this trend (cf. Figure 4b).

Weiner et al. (2001) model the stationary gas flow in the barred spiral NGC 4123, using a potential derived from an optical image, and find that the best fit to the velocity data requires a maximum disk model for the mass distribution. Lindblad et al. (1996) find likewise a relatively good fit for the bright barred spiral NGC 1365 with a maximum disk model. Figure 4 suggests that faster rotators have rounder disks, which are more self-gravitating. 
A view not necessarily in contradiction is voiced by Courteau et al. (2003), who contend that on average, disks with $\mathrm{V}_{\max }<200 \mathrm{~km} / \mathrm{s}$ are sub-maximal. They argue this on the basis of velocity dispersion data from Bottema (1997) [which I deem to have too large error bars], work on disk stability by Fuchs [but see Fuchs (2003)], the absence of the expected correlated scatter in the TullyFisher relation (Courteau \& Rix 1999) [but disk maximality seems to depend on $\left.\mathrm{V}_{\text {max }}\right]$, and the result on the lens $2237+0305$. Nevertheless, they find that TullyFisher relations for barred and un-barred galaxies are similar, in agreement with previous work, so barredness does not affect maximality.

Trott \& Webster (2002) combine for $2237+0305$ their lens model with HI rotation data further out. There is little need for a dark halo in the central parts, which are dominated by a bulge-bar system. Their statement that the disk is not maximal is partly influenced by their inclusion of the bar into the bulge, even though bars are thought to originate in the disk. For our own Galaxy, data on the microlensing towards the bulge-bar system likewise suggest that dark matter does not dominate in the central parts (Bissantz \& Gerhard 2002).

\section{Dwarf spheroidals and other satellites}

The $\Lambda$ CDM simulations predict the presence of far more satellite galaxies than is observed around the Milky Way, and the concept of failed dwarfs has been advanced to rescue the "theory". In this picture, studies of high velocity clouds are debated, but their individual distances are quite uncertain, and they don't indeed seem to have stars (e.g. Simon \& Blitz 2002).

The dwarf spheroidal companions of the Milky Way are very important in another way : the smallest ones are dark matter dominated. New data on the Draco system (Kleyna et al. 2002) show that this galaxy is more extended than previously thought; Stoehr et al. (2002) produce a good fit with $\Lambda$ CDM. But for Ursa Minor there is a subpopulation of stars with low velocity dispersion. Such a cold clump will survive easily in a cored halo potential, but breaks up rather rapidly in a cusped halo potential (Kleyna et al. 2003).

Finally, modeling the trail of the Sagittarius dwarf galaxy (e.g. Ibata et al. 2001) suggests that the dark halo around the Milky Way is nearly spherical.

\section{Elliptical galaxies}

Stellar kinematics of elliptical galaxies have turned up only 3 unambiguous cases where dark matter is needed to fit the data: NGC 2434, NGC 7507 and NGC 7626 (Kronawitter et al. 2000), partly because the data is limited in radius to $1-2 R_{e}$. Planetary nebulae can now be used as a tracer further out, and is becoming an industry. Interestingly, constant $\mathrm{M} / \mathrm{L}$ models can explain the new data, though M/L values are rather high (e.g. Romanowsky et al. 2003).

For field ellipticals, evidence for dark matter does come from HI studies, which show for several galaxies a flat rotation curve from $\sim 0.3 \mathrm{R}_{\mathrm{e}}$ out to $5-$ $6 \mathrm{R}_{\mathrm{e}}$ (e.g. Oosterloo et al. 2002). This implies a global $(\mathrm{M} / \mathrm{L})_{\mathrm{B}}$ ratio $\sim 25$. This value is in good apparent agreement with a similar value found based on $\mathrm{X}$-ray studies. Yet the new Chandra and XMM data show a wealth of detail in the images of the X-ray gas of individual galaxies, so much so that one can 
question the validity of the hydrostatic equilibrium equation used to evaluate masses. A further complication is the contribution to the X-ray flux of low mass $\mathrm{X}$-ray binaries, occurring presumably in globular clusters. Indeed, Paolillo et al. (2003) argue that the core of the X-ray emission is associated with the stellar distribution (gas from mass loss of evolving stars), and that the extended X-ray emission traces really the group or cluster potential rather than the potential of the elliptical galaxy itself. This agrees with studies using globular clusters as a tracer, e.g. for NGC 4472 and M87, which show that there is a need for dark matter at larger radii there (see Kronawitter et al. 2000 and references therein).

A most interesting development is the use of lensing data, combined with more classical spectroscopy, to estimate the mass of ellipticals. A very good example of this is the study of the lens $0047-281$ by Koopmans \& Treu (2003), who find indications that: 1) the total density follows a power law with slope -1.9 ; 2) a constant $\mathrm{M} / \mathrm{L}$ model can be eliminated using velocity dispersion data; and 3) the inner dark matter slope can be as shallow as -1.1 . For some lenses, the flux ratios cannot be matched with a smooth potential, and therefore substructure needs to be added to the models to produce a fit (e.g. Bradac et al. 2002). This is seen as consistent with the $\Lambda$ CDM prediction.

Acknowledgments. I thank Lia Athanassoula for frequent discussions, and Erwin de Blok and Stacy McGaugh for fruitful joint work on LSB galaxies.

\section{References}

Athanassoula, E. 1984, Physics Reports, 114, 319

Athanassoula, E., Bosma, A., \& Papaioannou, S. 1987, A\&A, 179, 23

Bell, E., \& de Jong, R.S. 2001, ApJ, 550, 212

Bissantz, N., \& Gerhard, O. 2002, MNRAS, 330, 591

Böker, T., et al. 2002, AJ, 123, 1389

Bosma, A. 1978, PhD Thesis, University of Groningen

Bosma, A., Byun, Y.I, Freeman, K.C., \& Athanassoula, E. 1992, ApJ, 400, L21

Bottema, R. 1997, A\&A, 328, 517

Bradac, M., et al. 2002, A\&A, 388, 373

Courteau, S., et al. 2003, ApJ, 594, 208

Courteau, S., \& Rix, H.-W. 1999, ApJ, 513, 561

de Blok, W.J.G., \& Bosma, A. 2002, A\&A, 285, 816

de Blok, W.J.G., Bosma, A., \& McGaugh, S.S. 2003, MNRAS, 340, 657

Franx, M., \& de Zeeuw, P.T. 1992, ApJ, 392, L47

Fuchs, B. 2003, in Fourth International Workshop on the Identification of Dark Matter, eds. N.J.C. Spooner \& V. Kudryavtsev (Singapore: World Scientific), p.72

Fukushige, T., \& Makino, J. 2001, ApJ, 557, 533

Fukushige, T., Kawai, A., \& Makino, J. 2003, astro-ph/0306203

Garrido, O., Marcelin, M., Amram, P., \& Boulesteix, J. 2002, A\&A, 387, 821

Hayashi, E. et al. 2003, astro-ph/0310576 
Hoekstra, H., van Albada, T.S., \& Sancisi, R. 2001, MNRAS, 323, 453

Ibata, R., Lewis, G.F., Irwin, M., Totten, E., \& Quinn, T. 2001 ApJ, 551, 294

Kleyna, J.T., Wilkinson, M.I., Evans, N.W., Gilmore, G., \& Frayn, C. 2002, MNRAS, 330, 792

Kleyna, J.T., Wilkinson, M.I., Gilmore, G., \& Evans, N.W. 2003, ApJ, 588, L21

Koopmans, L.V.E., \& Treu, T. 2003, ApJ, 583, 606

Kranz, T., Slyz, A., \& Rix, H.-W. 2001, ApJ, 562, 164

Kranz, T., Slyz, A., \& Rix, H.-W. 2003, ApJ, 586, 143

Kronawitter, A., Saglia, R.P., Gerhard, O., \& Bender, R. 2000, A\&AS, 144, 53

Lindblad, P.A.B., Lindblad, P.O., \& Athanassoula, E. 1996, A\&A, 313, 65

Marchesini, D. et al. 2002, ApJ, 575, 801

Matthews, L.D., \& Wood, K. 2001, ApJ, 548, 150

McGaugh, S.S., Rubin, V.C., \& de Blok, W.J.G. 2001, AJ, 122, 2381

Moore, B., Quinn, T., Governato, F., Stadel, J., \& Lake, G. 1999, MNRAS, 310, 1147

Navarro, J.F., Frenk, C.S., \& White, S.D.M. 1996, ApJ, 462, 563

Navarro, J.F., Frenk, C.S., \& White, S.D.M. 1997, ApJ, 490, 493

Nicastro, F. et al. 2003, Nature, 421, 719

Oosterloo, T.A. Morganti, R., Sadler, E.M., Vergani, D., \& Caldwell, N. 2002, AJ, 123,729

Palunas, P.A., \& Williams, T.E.B. 2000, AJ, 120, 2884

Paolillo, M., Fabbiano, G., Peres, G., \& Kim, D.-W. 2003, ApJ, 586, 850

Romanowsky, A. et al. 2003, Science, 301, 1696

Schoenmakers, R.H.M, 1999, PhD Thesis, University of Groningen

Simon, J.D., \& Blitz, L. 2002, ApJ 574, 726

Simon, J.D., Bolatto, A.D., Leroy, A., \& Blitz, L. 2003, ApJ, 596, 957

Slyz, A., Kranz, T., Rix, H.-W. 2003, MNRAS (in press), astro-ph/0309597

Spergel, D. et al. 2003, ApJS, 148, 175

Stoehr, F., White, S.D.M., Tormen, G., \& Springel, V. 2002, MNRAS 335, L84

Swaters, R.A. 1999, PhD Thesis, University of Groningen

Swaters, R.A., Madore, B., van den Bosch, F.C., \& Balcells, M. 2003a, ApJ, 583, 732

Swaters, R.A., Verheijen, M.A.W., Bershady, M.A., \& Andersen, D.R. 2003b, ApJ, 587, L19

Tegmark, M. et al. 2003, astro-ph/0310723

Trott, C.M., \& Webster, R.A. 2002, MNRAS, 334, 621

van den Bosch, F.C., Robertson, B.E., Dalcanton, J.J, \& de Blok, W.J.G. 2000, AJ, 119, 1579

Verheijen, M.A.W. 1997, PhD Thesis, University of Groningen

Visser, H.C.D. 1980, A\&A, 88, 159

Walter, F., \& Brinks, E. 1999, AJ, 118, 273

Weiner, B., Sellwood, J.A., \& Williams, T.B. 2001, ApJ, 546, 931 


\section{Appendix: $21 \mathrm{~cm}$ Line Interferometry and the Dark Matter Problem}

[Reprinted from the Magellanic Times, Day 7, 22 July 2003 - the newspaper edited during the 25th General Assembly of the IAU in Sydney.]

"Write me a piece, Albert. I'd really appreciate it, I won't have time to do it myself." I am sitting opposite Seth Shostak, editor of the Magellanic Times, whom I have known for a long time, and known of for even longer. He and I owe a lot of intellectual indebtedness and gratitude to a quiet man who patiently taught us the principles of $21 \mathrm{~cm}$ HI interferometry, David Rogstad. Rogstad was one of the first to use a two element radio aperture synthesis interferometer to study the $21 \mathrm{~cm}$ line emission of nearby galaxies (he was LOC co-chair John Whiteoak's first student.)

Seth did his thesis on detailed synthesis observations of 5 late-type galaxies with the Owens Valley Radio Observatory (OVRO) twin element interferometer. Dave Rogstad moved for a few years to the Kapteyn Laboratorium in Groningen in the late sixties, where he guided a couple of students in various projects Henk Olthof on warps, Arnold Rots on data of NGC 6946 and IC 342, and me. He himself worked on his OVRO data of M101.

For me, it was my first research project. I had to read reams of charts to mark off phase zero measurements of fringes from the just completed Westerbork telescope. Rogstad taught me computing and offered valuable advice ("beware, if you start computing, you stop thinking.") After this, he gave me his $21 \mathrm{~cm}$ line data of M82 to reduce, along with all his programs on punched cards. I was enthusiastic about this, looked up the literature on this "exploding galaxy", and learned about the galaxy rotation work of Burbidge, Burbidge and Prendergast. The M82 HI data were interesting, and Dave encouraged me to speak about them at a YERAC (Young European Radio Astronomy Conference) in Dwingeloo.

Dave's work on M101 stimulated the young staff members in Groningen, led by Ron Allen, to push for a temporary receiver to do $21 \mathrm{~cm}$ line work on nearby galaxies with the Westerbork telescope - which was not explicitly constructed for this purpose. By the time this project was completed, I was fortunate enough to use it for my thesis work on a pilot HI study of spiral galaxies of various morphological types. That was more or less [considered] a backwater project, the main attention for $21 \mathrm{~cm}$ line work being the study of spiral structure of M51, M81 and M101 ("testing the density wave theory.")

Even so, I witnessed the debate about rotation curves in the outskirts of galaxies using HI data. I vividly remember a seminar by Mort Roberts, arguing that M31's rotation curve was flat, and all the young Turks of the Kapteyn lab arguing that he picked up signal of the main disk through the sidelobes of his single dish antenna (Ed Salpeter was subjected to a similar treatment a few years later [at the IAU Symposium 77 in Bad Münstereiffel] when he reported on his Arecibo data, which had some after-effects if his Annual Reviews autobiography is any guide). So $21 \mathrm{~cm}$ line interferometry was the creed.

My thesis work, with its basic result that the rotation curves of spiral galaxies of all morphological types stayed flat (or were even still rising) beyond the optical image, helped settle the debate of the presence of dark matter in the outer parts of spirals. I also was fortunate to be able to go to Palomar with Piet van der Kruit, to get $\mathrm{H} \alpha$ rotation data in the inner parts, and to obtain plates 
for surface photometry to determine the luminosity profile and the mass-to-light ratio as a function of radius.

But the questions remain - how was the dark matter debate conducted, who said what when and why, and who had the "correct" (i.e. present day accepted) picture first? This is not so easy as some people would have it. In Historical Development of Modern Cosmology (ASP conference series Vol. 252), I read two articles - one by Sidney van den Bergh and one by Jaan Einasto - and there seems to be little overlap. So there could be different stories, and different people were convinced at different times of the presence of the dark matter in spirals just like the various events and characters which made up the French Revolution. In any case, as far as the HI line rotation curves are concerned, I can only cite Vera Rubin et al.'s (1978) paper where she writes that "Mort Roberts and his collaborators deserve credit for first calling attention to flat rotation curves", credit he got only sparingly. What is clear now is that there is little need for a dark halo to fit the optical rotation curve data, a conclusion [again] reached in an ApJ paper as recently as 2000. Mort Roberts himself confided to me once that he thought that my thesis work vindicated his contention that rotation curves of giant spirals are flat. Ostriker and Peebles, who pushed the idea for dark halos, first in a 1973 paper on disk stability (a paper which nowadays cannot withstand close scrutiny), and then, with Yahil, in their classic 1974 paper on various indicators pointing to a linear increase of the mass of a giant galaxy with radius, cite for the rotation data the papers by Roberts and Rots (1973) and Rogstad and Shostak (1972).

Which brings me back to Seth Shostak. Contrary to my thesis, which I had printed in 500 copies, and with its handy format became quite in demand (my thesis advisor had his copy stolen from his office somewhere in the 1980s, he told me recently), Shostak's thesis (1971) is not widely available. I found a copy of it in the VLA library, so I started reading it carefully, and yes, it contained the flat rotation curve of NGC 2403, and its implication that the volume density of matter drops off as $\mathrm{r}^{-2}$ (mass rises linearly with radius, therefore). So he had it! Okay, only for one case, but even so; and it was a clean observation with an interferometer, so unlike Roberts' observation of M31 (the primary piece of evidence in the Roberts and Rots (1973) paper). Oh well, it is unclear to me sometimes how scientific evidence is accepted in certain circles. Sometimes one has a result, correct after all, but at a time too far ahead of the pack, which is not yet ready to absorb it.

In any case, the amusing part of Seth's thesis is in the very end. A brief acknowledgement to Gordon Stanley, then director of OVRO. A warm thanks to David Rogstad for his patient advice. And then this fantastic flight of fancy - "this thesis is dedicated to NGC 2403 and its inhabitants, to whom copies can be furnished at cost". No wonder this man is now working on SETI! He just wants to know why nobody came along thus far to claim a copy. If somebody from that galaxy had come along, Seth would have been the most famous man on earth, offered an autographed copy of his thesis for free, and arranged for a public lecture at the current General Assembly. John Whiteoak would have been happy to schedule that, and the Harbourside Auditorium would have been way too small! 